\title{
Changes in gain of horizontal vestibulo-ocular reflex during spaceflight
}

\author{
Gilles Clément $^{\mathrm{a}, \mathrm{b}, *}$, Scott J. Wood ${ }^{\mathrm{c}}$, William H. Paloski ${ }^{\mathrm{c}}$ and Millard F. Reschke \\ ${ }^{\mathrm{a}} \mathrm{KBR}$, Houston, USA \\ ${ }^{\mathrm{b}}$ Lyon Neuroscience Research Center, Bron, France \\ ${ }^{\mathrm{c}}$ Neuroscience Laboratories, NASA Johnson Space Center, Houston, USA
}

Received 8 March 2019

Accepted 21 May 2019

\begin{abstract}
.
BACKGROUND: The vestibulo-ocular reflex (VOR) is a basic function of the vestibular system that stabilizes gaze during head movement. Investigations on how spaceflight affects VOR gain and phase are few, and the magnitude of observed changes varies considerably and depends on the protocols used.

OBJECTIVE: We investigated whether the gain and phase of the VOR in darkness and the visually assisted VOR were affected during and after spaceflight.

METHODS: We measured the VOR gain and phase of 4 astronauts during and after a Space Shuttle spaceflight while the subjects voluntary oscillated their head around the yaw axis at $0.33 \mathrm{~Hz}$ or $1 \mathrm{~Hz}$ and fixed their gaze on a visual target (VVOR) or imagined this target when vision was occluded (DVOR). Eye position was recorded using electrooculography and angular velocity of the head was recorded with angular rate sensors.

RESULTS: The VVOR gain at both oscillation frequencies remained near unity for all trials. DVOR gain was more variable inflight and postflight. Early inflight and immediately after the flight, DVOR gain was lower than before the flight. The phase between head and eye position was not altered by spaceflight.

CONCLUSION: The decrease in DVOR gain early in the flight and after the flight reflects adaptive changes in central integration of vestibular and proprioceptive sensory inputs during active head movements.
\end{abstract}

Keywords: Vestibular nystagmus, visual fixation, visual-vestibular interaction, microgravity, vestibulo-ocular reflex

\section{Introduction}

The vestibulo-ocular reflex (VOR) works in conjunction with the visual system to help maintain a clear, stable image on the retina by producing eye movements that compensate for movements of the head. To effectively stabilize gaze, the VOR is mediated primarily by vestibular information that relies on appropriate interaction between the semicircular canals and the otoliths. The dynamic characteristics of the passively evoked VOR have been well docu-

${ }^{*}$ Corresponding author: Gilles Clément, KBR, 2400 East NASA Parkway, Houston TX 77058, USA. Tel.: +1 281244 5720;

E-mail: gilles.r.clement@nasa.gov. mented and are routinely examined by measuring the relationship between stimulus and response during transient velocity steps or sinusoidal oscillations over a range of frequencies [32]. However, responses to passive perturbations may not reflect the true nature of the system responses because during natural behavior we usually move both our eyes and our head together. During voluntary head movements, we receive additional proprioceptive information from neck muscles and inputs from the efference copy of the motor command [13]. This additional information informs the central nervous system of a self-generated movement and prevents the illusion of a shift in the visual environment increases, thereby increasing the efficiency of the stabilizing reflex [30]. 
On Earth, the otolith organs of the vestibular system help the central nervous system interpret the position of the head in space by detecting head tilt relative to gravity. In the absence of a gravitational reference during spaceflight, the static otolith signals are no longer effective. The integration of proprioception and vestibular cues, critical for optimizing gaze reflexes during active head movements, is likely altered due to unloading of both the head and otoliths. During return to a normal $1 \mathrm{~g}$ environment, otolith inputs are restored and proprioceptive information changes. A misinterpretation of the head's position in space could result in a misperception of gaze position, which would alter the VOR.

VOR has been studied in space during voluntary head oscillations at frequencies ranging from 0.25 to $1.0 \mathrm{~Hz}[1,21-23,26,38-41,43]$. Six hours into the Space Shuttle mission STS-51G, a crewmember exhibited a significant decrease in VOR gain in darkness during voluntary head oscillations in yaw at $0.25 \mathrm{~Hz}$; this decrease recovered to preflight levels by flight day 7 [41]. A decrease in yaw VOR gain early in flight is consistent with results from studies conducted in parabolic flight (20-25 seconds of microgravity) $[24,40]$. Since no phase shift accompanied the in-flight reduction in yaw VOR gain, the authors of the STS-51G investigation speculated that the subject might have suppressed vestibular input to avoid sensory conflict, possibly as a learned response from his prior training as a pilot [41]. However, it is also possible that the microgravity-induced changes in otolith and proprioceptive inputs affected the crewmember's ability to imagine a fixed visual target.

Decay in horizontal post-rotatory nystagmus was measured in 4 astronauts during Space Shuttle missions lasting 10-12 days by spinning the subjects on a rotating chair in darkness and then suddenly stopping the chair. No change was observed in the initial peak slow-phase velocity of the VOR; however, spaceflight reduced the time constant of post-rotatory nystagmus [28, 29]. Four astronauts who participated in a different short Space Shuttle mission also had a shorter time constant of the post-rotatory nystagmus than baseline values during their first flight day and after they returned from space, but they had no consistent change in the magnitude of the initial peak slow-phase velocity response [27]. These effects are qualitatively similar to effects observed in parabolic flight [16, 17]. A decrease in the time constant of nystagmus was also observed in 2 monkeys after they returned from space [10], and this decrease was associated with a postflight decrease in activity of the primary afferents in their vestibular end organ [12].

The time constant of nystagmus gives an evaluation of velocity storage [33], which is defined as the nystagmus response to a velocity step that outlasts the physical return of the cupula-endolymph system of the semicircular canals to resting levels. Velocity storage is thought to reflect the midbrain integration of a velocity signal originating from the semicircular canals. When the otolith organs are ablated, velocity storage is abolished [11], which suggests that the velocity storage mechanism is sensitive to linear acceleration and gravity. Studies have shown a modulation of the yaw VOR due to changes in the otolith cues mediated by velocity storage [25] and that the three-dimensional organization of velocity storage was influenced by gravity [34]. During head movements in pitch and roll, the vertical semicircular canals are stimulated by angular acceleration, and the otolith organs are stimulated by head tilt relative to gravity. A decrease in the gain of the vertical and torsional VOR during and after spaceflight has been well documented [2-4, 8, 18, 21-23, 41]. Such a decrease is expected is microgravity because the otoliths are no longer contributing to signaling head tilt during pitch and roll head movements. When the head upright is rotated in yaw in normal gravity, the otoliths are not stimulated by the head movements, but they still signal the head's orientation relative to gravity. This function is absent in microgravity; therefore it is possible that spaceflight could also affect the horizontal VOR gain and phase.

Previous attempts to determine how spaceflight affects horizontal VOR gain were limited by the relatively short duration of the spaceflights and the limited opportunities to measure these effects early in the flight and shortly after return. The purpose of the present study was to measure horizontal VOR gain and phase at regular intervals during Space Shuttle flights. Subjects performed voluntary sinusoidal head movements at a low $(0.33 \mathrm{~Hz})$ and high frequency $(1.0 \mathrm{~Hz})$ in two visual conditions: with their gaze fixed on a visual target (VVOR) or while attempting to fix their gaze on the target while their eyes were open but covered (DVOR). Measurements were obtained throughout the flight and within 2 hours of landing, and we evaluated the difference in gain and phase between the two visual conditions. 


\section{Material and methods}

\subsection{Subjects}

Four male astronauts participated in this study. All other information about flight and subject demographics has been withheld to protect identification. All subjects passed the required medical flight certifications and had no known neurological, vestibular or sensory-motor abnormalities were present in any of the participating crewmembers. All subjects gave their informed consent for inclusion before they participated in the study. The study was conducted in accordance with the Declaration of Helsinki, and the protocol was approved in advance by the National Aeronautics and Space Administration Institutional Review Board.

\subsection{Experiment schedule}

The subjects were trained to perform the protocol, and baseline data were then collected 60, 30, and 15 days (L-60, L-30, and L-15) before the launch. Inflight data were obtained on flight days (FD) 2, 5, 10, and 16. Postflight data collection began approximately 2 hours after the landing of the Space Shuttle $(R+0)$, and was followed up with additional data collection sessions on $R+1, R+2, R+4$, and $R+8$ days. Due to either scheduling or time constraints, not all subjects were tested for each visual condition on every test day. Table 1 summarizes the tests completed by each subject.

\subsection{Experimental protocol}

The subjects actively oscillated their head relative to their trunk around the long body axis (yaw) in a

Table 1

Test schedule and visual conditions for the four astronauts (A, B, C, D)

\begin{tabular}{lcc}
\hline Test Day & VVOR & DVOR \\
\hline L-60 & A, B, C, D & A, B, D \\
L-30 & A, B, C, D & A, B, C, D \\
L-15 & A, B, C, D & A, B, C, D \\
FD2 & A, B, C, D & B, C, D \\
FD5 & B, C & B, C \\
FD10 & A, B, C, D & A, B, C, D \\
FD16 & A, B, C, D & A, B, C, D \\
R+0 & & A, B, C, D \\
R+1 & A, B, C, D & A, B, C, D \\
R+4 & A, B, C, D & A, B, C, D \\
R+8 & A, B, C, D & A, B, C, D \\
\hline
\end{tabular}

sinusoidal fashion to determine the relative contributions of the VOR when their vision was occluded (DVOR), or in conjunction with the smooth pursuit system for maintaining gaze stability (VVOR). In the VVOR condition the fixed target was visible at all times. In the DVOR condition the subject first fixed their gaze on the target, then his vision was occluded with light-tight goggles, and he oscillated his head while fixing his gaze on the remembered location of the target.

The visual target was a red LED in the center of a cruciform target display (Fig. 1) that was placed $1 \mathrm{~m}$ in front of the subject. All targets subtended a visual angle of approximately $1^{\circ}$. The subjects sat with their legs and trunk tightly restrained. Sound cues for the sinusoidal head oscillation were provided by a $2 \mathrm{kHz}$ carrier tone that was modulated in pitch at the primary frequencies of $0.33 \mathrm{~Hz}$ and $1.0 \mathrm{~Hz}$. Subjects were instructed to move their head to the left in response to the low pitch of the modulated frequency and to smoothly generate sinusoidal head movements such that when the modulated tone reached its highest pitch the head would be at its peak rightward displacement. No attempt was made to regulate the amplitude of the head movements by either training or feedback. By following the auditory cue, the subjects made smooth head transitions from the low frequency $(0.33 \mathrm{~Hz})$ to the high frequency $(1.0 \mathrm{~Hz})$. With limited practice, all subjects were capable of executing sinusoidal head movements that followed the auditory cues. The responses to the first five cycles for each of the individual tones were excluded from our analysis. Data from the final 5 to 10 cycles were then averaged to estimate the response parameters of peak-to-peak amplitude and phase relationships.

\subsection{Eye movements}

Eye position was recorded using standard electrooculography (EOG). Surface electrodes were placed at the outer canthi of each eye to record the horizontal eye movements and the neutral electrode was affixed over the right temporal bone. The horizontal eye position was calibrated using the horizontal LED targets on the cruciform target display just prior to performing the head oscillations. During the calibration, subjects were instructed to look at the LED at the center of the display (the same target used later for visual fixation) and then to look at targets located $\pm 10^{\circ}, \pm 20^{\circ}$ and $\pm 30^{\circ}$ off-center. Calibration curves were generated by plotting the EOG signals corre- 

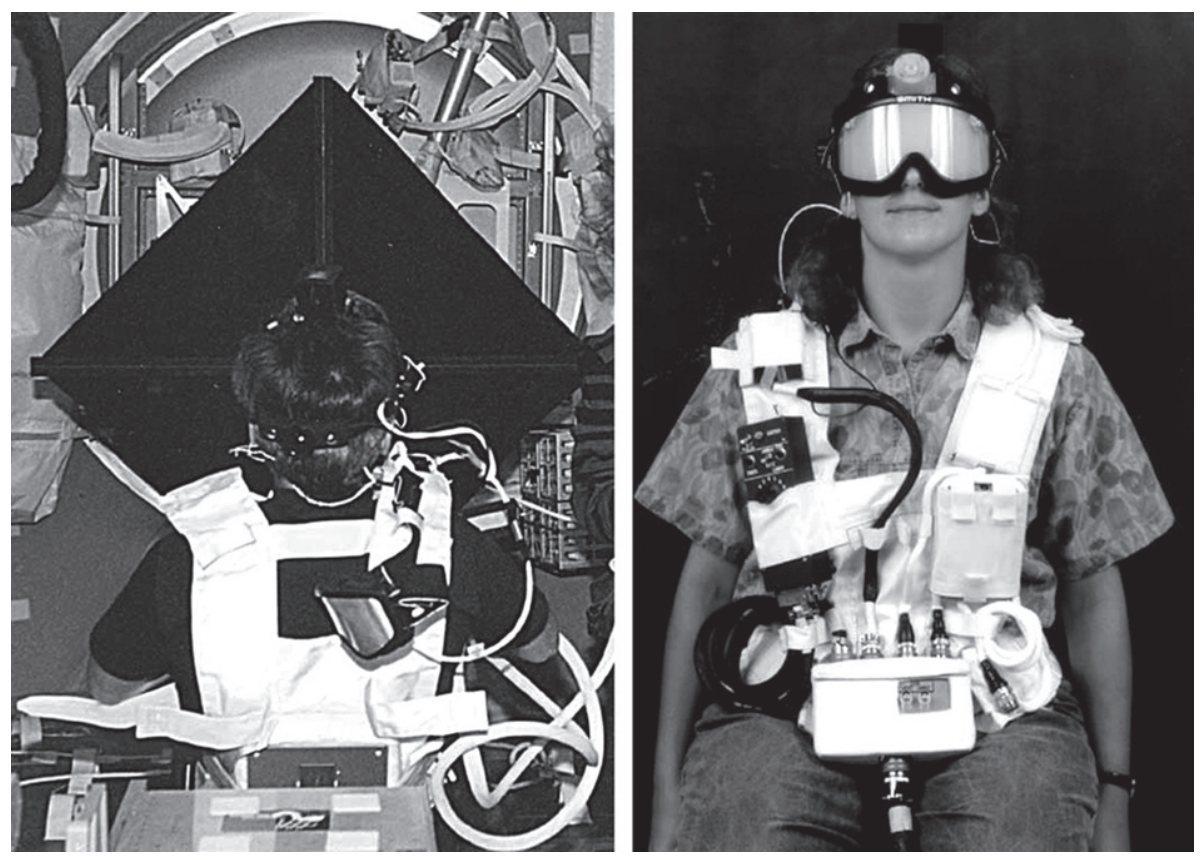

Fig. 1. Left. Photograph showing a crewmember performing the experiment on board the Space Shuttle. The rate sensors housing unit is seen on the top of subject's head. Directly in front of the subject is the cruciform target display used for head and eye calibrations, and for the presentation of the central target during head oscillations. Right. Photograph showing the head-mounted laser for head position calibration and the goggles that can occlude the subject's vision. The black box is the controller used by the operator to control the visual display, the goggles occluding, and the cassette tape recorder that plays the frequency-modulated tones used for pacing the head oscillations. Photos credit: NASA.

sponding to each displacement, and a best linear fit was determined using least-squares techniques.

Signals from the electrodes were amplified (4K) over a frequency ranging from DC to $35 \mathrm{~Hz}$. When the eye drift exceeded the amplifier limits, the eye signal was returned to a balanced offset with a DC bias restoring circuit. A six-pole low pass active Bessel filter was used to prevent aliasing of the data. Filtered data were sampled at $500 \mathrm{~Hz}$ and post-processed signals were passed through a $15 \mathrm{~Hz}$ digital low pass Chebyshev filter prior to analysis.

\subsection{Head movements}

A tri-axial angular rate gyro (Watson Industries, Inc., Eau Claire, WI, USA) mounted on a headband measured head angular velocity about the yaw, pitch, and roll axes. Before each session, angular rate signals were calibrated using a head-mounted laser and the $0^{\circ}$ and $30^{\circ}$ calibration light emitting diodes (LEDs) on the target display. While wearing the head laser, the subjects were cued to move their head as accurately they could from the center LED to the $30^{\circ}$ rightward LED. When the rightward LED was extinguished the subjects were cued to move their head back to the center LED. The procedure was repeated for both the leftward and vertical targets. Calibrations were performed a minimum of 2 times before data collection began.

\subsection{Data analysis}

The rate sensor data were integrated to obtain head position. Component crosstalk signals from the 3 different angular rates (yaw, pitch, roll) were nulled before analysis to exclude difference in sensor mounting. This nulling was obtained by generating a rotation matrix using data from the calibration trials for horizontal (yaw) and vertical (pitch) movements of the head. We assumed that the head-mounted laser visually guided the subjects to generate planar motion, and that any incidental out-of-plane velocity components would have been balanced with motion in the opposite direction during the head calibration. Although we did not perform calibrations of head movement in roll, we assumed that the angular rate sensors were mounted in orthogonal planes with respect to one another. Once the rotation matrix had been determined it was applied to all trials to minimize the effects of unwanted crosstalk. 
Our primary interest was to evaluate the VOR gain and phase under different visual and gravitational conditions and to determine how this affects fixation of gaze on a stationary target. Optimal curve-fitting techniques for position and velocity waveforms were used to obtain measures of VOR gain and phase, and gaze error with respect to the target. Sinusoidal curve fits to the subject-generated head motions were obtained based on the equation:

$$
r(t)=B r+A r \sin (2 \pi f+\emptyset r)
$$

where $B r$ is response bias, $A r$ is response amplitude, $f$ is the stimulus frequency, and $\emptyset r$ is response phase in degrees. The frequency that the eye position and velocity fit was defined by the stimulus (head) frequency, and the head signal was analyzed separately to determine the stimulus amplitude $(A s)$ and phase (Øs). The gain was then defined by the ratio Ar/As, and the phase by $\emptyset r-\varnothing_{s}+180^{\circ}$ so that a compensatory response had a gain of 1 and phase of $0^{\circ}$ [32]. There was little difference between the gains calculated with either the position or velocity data. However, velocity signals were noisier than the position signals. For this reason, all gain and phase were calculated using eye and head position (peak-to-peak amplitude) after integrating the rate sensor data obtained from the head movements.

\subsection{Statistical analysis}

For purposes of statistical analysis, the average gain and phase shift for each experimental unit (subject $\times$ flight day) was calculated over both the $0.33 \mathrm{~Hz}$ and $1.0 \mathrm{~Hz}$ frequencies. To avoid questionable assumptions of normality or homogeneity of variance in the eye movement analysis, all statistical inference on the effect of flight, differences in vision (VVOR; DVOR); and the interaction between vision and flight was made using the Wilcoxon Signed Rank test for paired data or the Wilcoxon Rank Sum test for unpaired data [37].

\section{Results}

Figure 2 shows examples of recordings of one subject's head position when he was cued with a modulate tone to oscillate his head at a $0.33 \mathrm{~Hz}$ and a $1.0 \mathrm{~Hz}$. The power spectral analysis of the head position indicates that the subject was able to match the frequency of the modulated tone almost perfectly.
Figure 3 shows the mean peak-to-peak amplitude for all subjects of head movements around the yaw, pitch, and roll axes obtained by integrating the angular rate sensor signals. A repeated measure ANOVA with two factors (visual conditions: VVOR, DVOR; sessions: L-60 to $R+8$ ) indicated no significant differences in head yaw amplitude at $0.33 \mathrm{~Hz}$ across visual conditions [F (1, $87)=2.63, p=0.109]$ and sessions $[\mathrm{F}(10,87)=1.93$, $p=0.06]$. There were also no significant differences in head yaw amplitude at $1.0 \mathrm{~Hz}$ across visual conditions $[\mathrm{F}(1,87)=3.25, p=0.07]$ and sessions $[\mathrm{F}$ $(10,87)=1.41, p=0.19]$. For pitch head movements, the ANOVA indicated no significant difference in amplitude between VVOR and DVOR at $0.33 \mathrm{~Hz}[\mathrm{~F}$ $(1,87)=0.36, p=0.55]$ and at $1.0 \mathrm{~Hz}[\mathrm{~F}(1,87)=0.12$, $p=0.73]$. However, there was a significant difference in head pitch amplitude at $0.33 \mathrm{~Hz}$ across sessions $[\mathrm{F}(10,87)=3.67, p<0.001]$, but not at $1.0 \mathrm{~Hz}[\mathrm{~F}$ $(10,87)=0.92, p=0.52]$. There were no significant differences in head roll amplitude across visual conditions and frequencies $[\mathrm{F}(3,175)=0.08, p=0.96]$ and test sessions $[\mathrm{F}(10,175)=1.63, p=0.10]$. Before the flight, the amplitude of head movements during both visual conditions and at both frequencies were $55.3^{\circ} \pm 5.4^{\circ}$ in yaw, $1.8^{\circ} \pm 0.6^{\circ}$ in pitch, and $9.9^{\circ} \pm 2.2^{\circ}$ in roll (mean $\pm \mathrm{SD}$ ). On flight day 2 , the amplitude of head movements in pitch increased $\left(5.9^{\circ} \pm 0.3^{\circ}\right)$ compared to preflight values. Student $t$-test indicated that this difference was significant $(p=0.01)$.

The VOR gains during both visual conditions and at both frequencies were similar for all 3 preflight tests (Fig. 4). No VVOR gain measurements were made on $R+0$ due to crew time constraints; however, the mean VVOR gain at both oscillation frequencies was near unity before, during, and after the flight (Fig. 4A,B). Before the flight, the mean DVOR gain was near unity during head movements at $0.33 \mathrm{~Hz}$ (Fig. 4C) and DVOR gain ranged between 0.92-0.94 at $1.0 \mathrm{~Hz}$ (Fig. 4D). From flight day 2 (i.e. 30 hours into the mission) to flight day 10 , the DVOR gain at $1 \mathrm{~Hz}$ decreased (mean value of 0.78 ) in comparison to baseline levels, and partially recovered by flight day 16 (Fig. 4D); DVOR gain was still lower than baseline immediately after landing $(R+0)$, and returned to preflight levels one day later. The DVOR gain at $0.33 \mathrm{~Hz}$ also decreased early in the flight and immediately postflight (Fig. 4C); however, the magnitude of the changes was less than the changes for $1.0 \mathrm{~Hz}$.

Spaceflight had little or no effect on the VVOR gain for either of the head oscillation frequencies we 

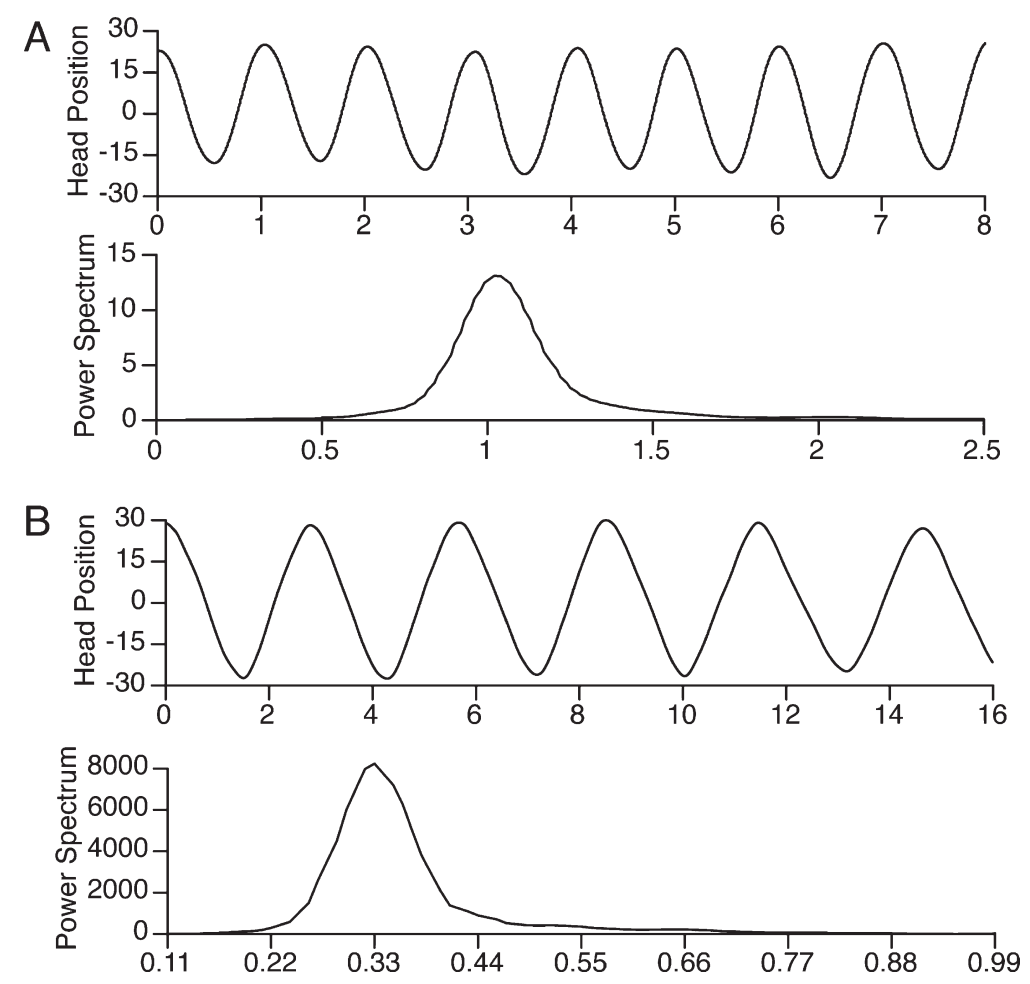

Fig. 2. Examples of head position recordings as a function of time accompanying a $0.33 \mathrm{~Hz}$ modulated tone (A) and a $1.0 \mathrm{~Hz}$ modulated tone (B) in the VVOR condition and the corresponding power spectral analyses. X-axis units are s for head position and Hz for power spectrum. Y-axis units are degrees.

tested (Table 2). By contrast, the DVOR gain during the flight was reduced for both $1.0 \mathrm{~Hz}(-10.9 \%$; $p=0.0084$, Wilcoxon Rank Sum test) and $0.33 \mathrm{~Hz}$ $(-4.4 \% ; p=0.087)$ compared to preflight. Although the preflight DVOR gain was lower at $1.0 \mathrm{~Hz}$ than at $0.33 \mathrm{~Hz}(-7.5 \%)$, the difference was far greater during the flight $(-15.4 \%)$, resulting in a significant interaction ( $p=0.028$, Wilcoxon Rank Sum test). At $0.33 \mathrm{~Hz}$, there was no difference between VVOR and DVOR gains during the preflight period, but there was a suggestion of interaction, manifested by a modest decrease in DVOR gain during the flight $(p=0.078)$, whereas the VVOR gain remained constant. A comparison of postflight and preflight results indicated that the effect of spaceflight on DVOR gain was still present immediately after return from space $(p=0.05$ at $0.33 \mathrm{~Hz} ; p=0.004$ at $1.0 \mathrm{~Hz}$ ).

When viewing the target (VVOR) before the flight, the subjects' head and eyes were almost in phase during both oscillation frequencies (Fig. 5A,B). However, during the trials when vision was occluded (DVOR) there was a slight positive phase shift, i.e., the eye movement was leading the head movement (Fig. 5C,D). Spaceflight had no significant effect on phase shift in the VVOR condition. The positive phase shift in the DVOR condition increased during flight at both oscillation frequencies, and the magnitude of increase was similar throughout the duration of the flight. Nevertheless, the median difference between the DVOR and VVOR phase shifts was higher than baseline during the flight $(p=0.054$, Wilcoxon Rank Sum test), indicating a likely interaction.

\section{Discussion}

The results of this investigation support the hypothesis that spaceflight affects the vestibular responses to self-rotation of the head in space relative to a stationary trunk, especially in the absence of vision. The VOR is mediated by the semicircular canals of the inner ear, which are activated by angular acceleration; by the otolith organs, which are sensitive to gravitoinertial acceleration; and (to a lesser extent) by neck proprioceptive inputs. The integration of canal, otolith, and proprioceptive cues is critical for optimizing gaze reflexes during active head movements. In 

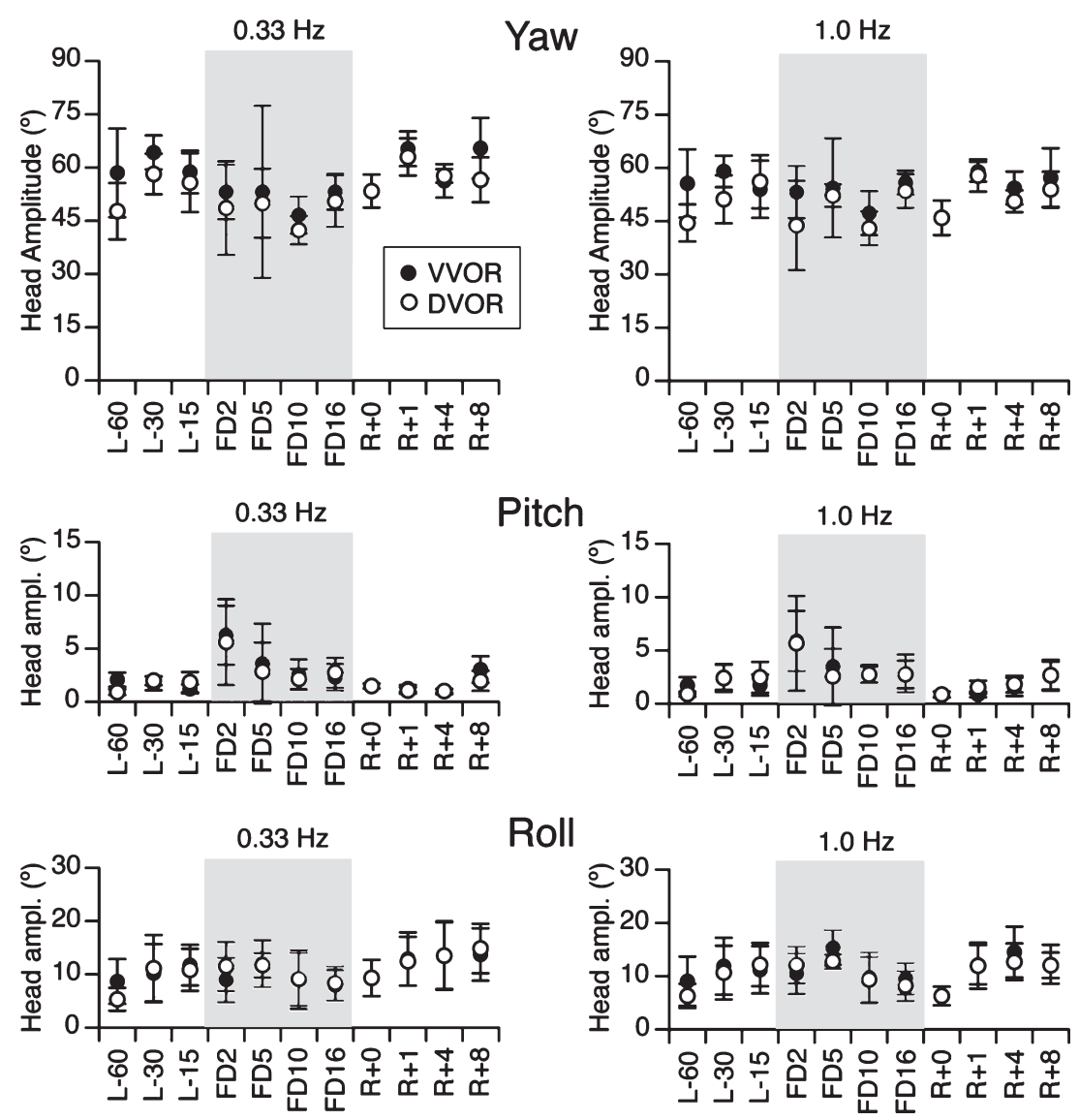

Fig. 3. Peak-to-peak amplitude of head movements about the yaw, pitch, and roll axes for both oscillation frequencies and visual conditions. Note that the head movements were predominantly in the yaw plane throughout all flight phases. Mean \pm SD of all subjects.

microgravity, this integration is altered and the resulting modification of the VOR can cause retinal slip and possible degradations in visual performance.

As would be expected in a simple goal-oriented task such as visual fixation during predictable head movements, VOR gain is mostly effective during lower frequency $(0.33 \mathrm{~Hz})$ head oscillation when vision is present (VVOR). However, at high frequency $(1.0 \mathrm{~Hz})$ head oscillation, the relatively slow response of the pursuit system begins to fail and vestibular information primarily drives stabilization of the retinal image. In microgravity, the otoliths have less influence on the velocity storage mechanism $[10,15]$; consequently, microgravity affects VOR more during faster head movements. However, vision appears to compensate for this change in vestibular function in microgravity, at least at the frequencies of head oscillations we tested in the current study.

Our results indicate that an astronaut's performance would not be compromised under normal visual conditions. However, when an astronaut performs tasks in low ambient light, or with unstructured vision or other off-nominal visual conditions, a decrease in DVOR gain, could negatively affect their gaze. This is particularly true if the change in DVOR gain is accompanied by a phasic change where the eye leads the head, as shown in our in-flight data. This gaze error could explain the oscillopsia experienced by some astronauts, and the changes in their perception of self- or surround-motion that are evoked during head movements $[35,36]$.

The amplitudes of yaw head movements were approximately constant across conditions and test sessions. The amplitude of pitch head movements at $0.33 \mathrm{~Hz}$, however, increased significantly when initially in space, even in the target-viewing condition (VVOR). This could represent difficulty in motor control (maintaining head-neck posture) or deficient processing of vestibular information to maintain orientation. The pitch movements might also, through 

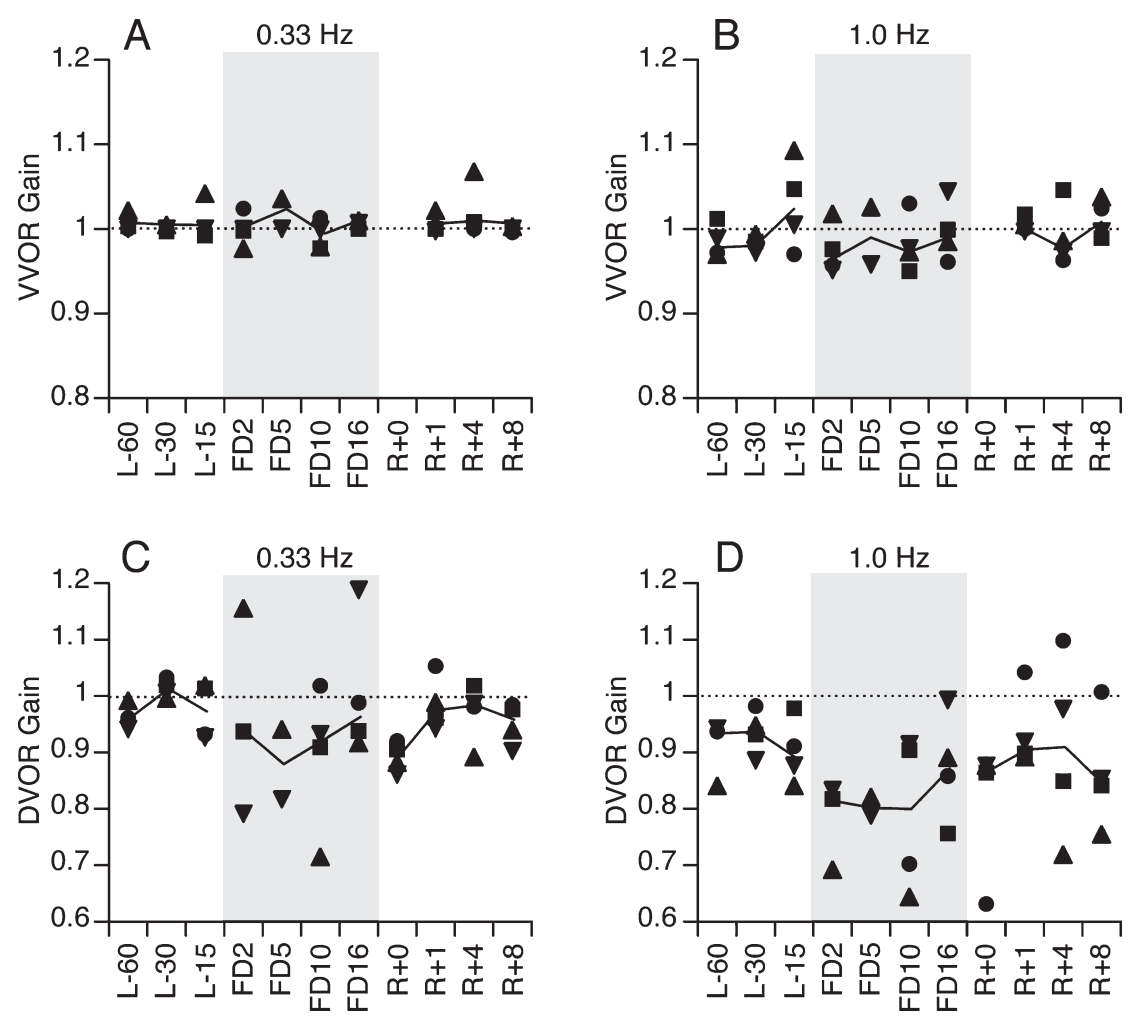

Fig. 4. VVOR (A,B) and DVOR (C,D) gain during head oscillations at $0.33 \mathrm{~Hz}$ and $1.0 \mathrm{~Hz}$ as a function of flight day. Symbols represent individual subject's values and continuous line is the median gain.

interaction with yaw movements, contribute to the reduction in DVOR gain in flight compared to preflight. Determining the extent to which vertical eye movements associated with this pitching contributed to the reduction in DVOR gain was limited by the EOG recording used.

It is well known that the eyes diverge when vision is occluded; in the dark, eyes focus at approximately $2 \mathrm{~m} \mathrm{[42].} \mathrm{It} \mathrm{is} \mathrm{possible} \mathrm{that} \mathrm{our} \mathrm{subjects} \mathrm{would} \mathrm{have}$ had difficulty maintaining the same eye convergence when vision was occluded as they did when viewing the target. Previous studies have measured changes in vergence eye movements in microgravity $[7,14]$. A change in fixation distance during the DVOR task could affect the gain since the eyes are not aligned with the axis of yaw rotation. If vergence reverted to the more distant dark focus rather than the actual target distance $(1 \mathrm{~m})$, then a lower amplitude of the eye would be required to stabilize this target, and the DVOR gain would decrease [20]. Based on a radius of the eyes at $0.08 \mathrm{~m}$ from the axis of yaw rotation [31], an average interpupillary distance of $60 \mathrm{~mm}$, and head movement amplitude of $\pm 50^{\circ}$, we calculated the horizontal gain would be expected to decrease
Table 2

Mean values of VVOR and DVOR gains and their difference (VVOR-DVOR) for both frequencies preflight, in flight, and postflight. Results of the Wilcoxon Signed Rank test for zero median are indicated by asterisks

\begin{tabular}{lcccc}
\hline Frequency & Test & VVOR & DVOR & Difference $(\%)$ \\
\hline $1.0 \mathrm{~Hz}$ & Preflight & 0.999 & 0.916 & $8.3^{* *}$ \\
& In flight & 0.986 & 0.816 & $17.2^{* *}$ \\
& Postflight & 1.003 & 0.889 & $11.4^{*}$ \\
$0.33 \mathrm{~Hz}$ & Preflight & 1.005 & 0.985 & 2.0 \\
& In flight & 1.002 & 0.942 & 6.0 \\
& Postflight & 1.008 & 0.950 & $5.8^{*}$ \\
\hline
\end{tabular}

${ }^{*} P<0.05 ;{ }^{* *} P<0.01$.

approximately $3 \%$ from a fixation of $1 \mathrm{~m}$ to $2 \mathrm{~m}$. This may contribute to the $10.9 \%$ and $4.4 \%$ decrease in DVOR gain in flight compared to preflight at $1.0 \mathrm{~Hz}$ and $0.33 \mathrm{~Hz}$, respectively. This may also contribute in part to the lower gains in the preflight tests when vision was absent.

Another contributing factor for the reduced DVOR gain in flight and early postflight could be due the astronauts' spatial disorientation in microgravity. Previous studies have shown that perception of distance and depth is altered during transitions between 

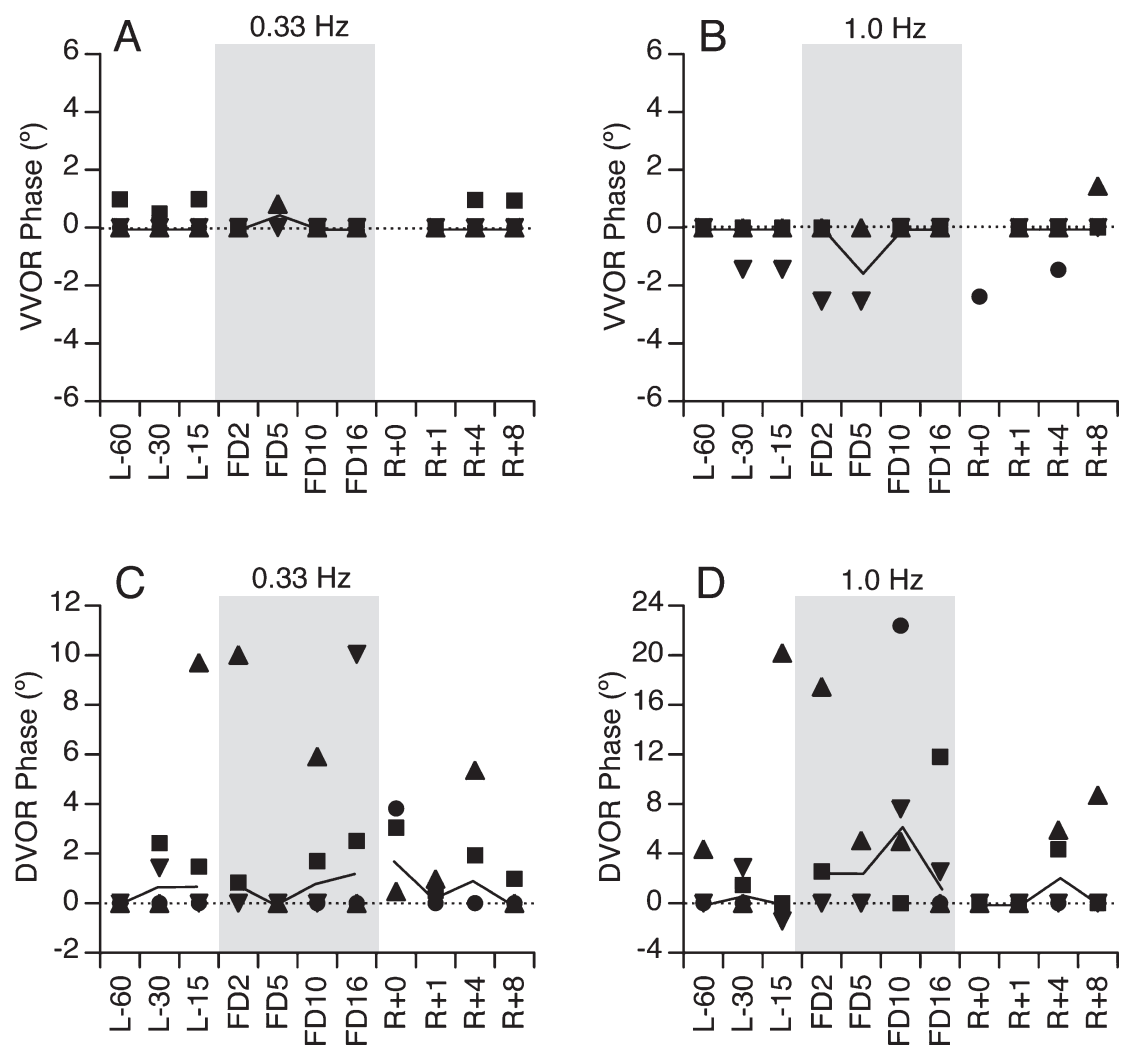

Fig. 5. VVOR (A,B) and DVOR (C,D) phase during head oscillations at $0.33 \mathrm{~Hz}$ and $1.0 \mathrm{~Hz}$ as a function of flight day. Symbols represent individual subject's values and continuous line is the median gain.

gravitational states $[5,6,9]$. It is possible that spatial disorientation makes it more difficult to imagine the location of a fixed target. In addition, the lower DVOR gain in flight compared to preflight values could be because the subject is suppressing vestibular information to reduce the confusing spatial orientation information.

Given the cost and difficulty of installing a servocontrolled rotating chair on board a spacecraft, active head oscillation appears to be a reasonable alternative method of investigating visual-vestibular function to different gravitoinertial environments [19]. The present study needs to be expanded to include additional oscillation frequencies to fully define the characteristics of the vestibulo-ocular transfer function. In addition to head oscillations, gaze stabilization trials (where the head is rotated actively with a velocity ramp-like profile), and head impulse testing (where the head is rotated passively at small amplitude and high acceleration) could also be performed. Astronauts must also be assessed as early as possible when they arrive in space and throughout long-duration missions if we are to accurately characterize the effects of spaceflight on gaze performance during visual-vestibular interaction.

\section{Conflicts of interest}

The authors declare that no conflicts of interest exist.

\section{Acknowledgments}

The authors thank the crewmembers for their participation in this experiment; Dr. Donald E. Parker (now deceased) for his contribution to experiment design; Pierre Simon (now deceased) for making the inflight hardware available for our use; Jenny Krug and Jody Cerisano for their assistance in data analysis; and Kerry George for her editorial suggestions. This study was supported by the National Aeronautics and Space Administration (NASA). 


\section{References}

[1] A.J. Benson and T. Viéville, European vestibular experiments on the Spacelab-1 mission: 6. Yaw axis vestibuloocular reflex, Exp Brain Res 64 (1986), 279-283.

[2] A. Berthoz, T. Brandt, J. Dichgans, T. Probst, W. Bruzek and T. Viéville, European vestibular experiments on the Spacelab-1 mission: 5. Contribution of the otoliths to the vertical vestibulo-ocular reflex, Exp Brain Res 64 (1986), $272-278$.

[3] A.H. Clarke, J. Grigull, R. Mueller and H. Scherer, The three-dimensional vestibulo-ocular reflex during prolonged microgravity, Exp Brain Res 134 (2000), 322-334.

[4] A.H. Clarke, H. Scherer and J. Schleibinger, Evaluation of the torsional VOR in weightlessness, J Vestib Res 3 (1993), 207-218.

[5] G. Clément, H.C.M. Allaway, M. Demel, A. Golemis, A.N. Kindrat, A.N. Melinyshyn, T. Merali and R. Thirsk, Long-duration spaceflight increases depth ambiguity of reversible perspective figures, PLoS One 10(7) (2015), e0132317.

[6] G. Clément and S.J. Wood, Rocking or rolling - Perception of ambiguous motion after returning from space, PLoS One 9(10) (2014), e111107.

[7] G. Clément and S.J. Wood, Translational otolith-ocular reflex during off-vertical axis rotation in humans, Neurosci Lett 616 (2016), 65-69.

[8] G. Clément, S.J. Wood, M.F. Reschke, A. Berthoz and M. Igarashi, Yaw and pitch visual-vestibular interaction in weightlessness, J Vestib Res 9 (1999), 207-220.

[9] G. Clément, A. Skinner and C.E. Lathan, Distance and size perception in astronauts during long-duration spaceflight, Life 3 (2013), 524-537.

[10] B. Cohen, I. Kozlovskaya, T. Raphan, D. Solomon, D. Helwig, N. Cohen, M. Sirota and S. Yakushin, The vestibulo-ocular reflex (VOR) of rhesus monkeys after spaceflight in the COSMOS biosatellite 2044, J Appl Physiol (1985) 73 (1992), S121-S131.

[11] B. Cohen, J.L. Suzuki and T. Raphan, Role of the otolith organs in generation of horizontal nystagmus: Effects of selective labyrinthine lesions, Brain Res 276 (1983), 159-164.

[12] M.J. Correia, A.A. Perachio, J.D. Dickman, I.B. Kozlovskaya, M.G. Sirota, S.B. Yakushin and I.N. Beloozerova, Changes in monkey horizontal semicircular canal afferent responses after space flight, $J$ Appl Physiol (1985) 73 (1992), 112S-120S.

[13] K.E. Cullen, Sensory signals during active versus passive movement, Curr Opin Neurobiol 14 (2004), 698-706.

[14] M. Dai, T. Raphan, I. Kozlovskaya and B. Cohen, Modulation of vergence by off-vertical yaw axis rotation in the monkey: Normal characteristics and effects of space flight, Exp Brain Res 111 (1996), 21-29.

[15] P. DiZio and J.R. Lackner, Influence of gravitoinertial force level on vestibular and visual velocity storage in yaw and pitch, Vision Res 32 (1992), 123-145.

[16] P. DiZio and J.R. Lackner, The effects of gravitoinertial force level and head movements on post-rotational nystagmus and illusory after-rotation, Exp Brain Res 70 (1988), 485-495.

[17] P. DiZio, J.R. Lackner and J.N. Evanoff, The influence of gravitoinertial force level on oculomotor and perceptual responses to sudden stimulation, Aviat Space Environ Med 58 (1987), A224-A230.
[18] V.K. Grigorova and L.N. Kornilova, Microgravity effect on the vestibulo-ocular reflex is dependent on otolith and vision contributions, Aviat Space Environ Med 67 (1996), 947-954.

[19] T.P. Hirvonen, H. Aalto, I. Pyykkö and M. Juhola, Comparison of two head autorotation tests, J Vestib Res 9 (1999), $119-125$.

[20] W.P. Huebner, M.F. Reschke, W.H. Paloski, J.J. Bloomberg, D.L. Harm and A. Berthoz, Dealing with the geometric effects of eye eccentricity in processing horizontal and vertical eye and head movement data, J Vestib Res 5 (1995), 299-322.

[21] L.N. Kornilova, V. Grigorova and G. Bodo, Vestibular function and sensory interaction in space flight, $J$ Vestib Res 3 (1993), 219-230.

[22] L.N. Kornilova, I.A. Naumov, S. Zarovska and V.N. Agalovitch, Gaze control and vestibular-cervical-ocular responses after prolonged exposure to microgravity, Aviat Space Environ Med 83 (2012), 1123-1134.

[23] L.N. Kornilova, S.V. Sagalovitch and V.V. Temnikova, Static and dynamic vestibulo-cervico-ocular responses after prolonged exposure to microgravity, J Vestib Res 17 (2007), 217-226.

[24] J.R. Lackner and A. Graybiel, Variations in gravitoinertial force level affect the gain of the vestibulo-ocular reflex: Implications for the etiology of space motion sickness, Aviat Space Environ Med 52 (1981), 154-158.

[25] R.F. Lewis, C. Haburcakova, W. Gong, F. Karmali and D.M. Merfeld, Spatial and temporal properties of eye movements produced by electrical stimulation of semicircular canal afferents, J Neurophysiol 108 (2012), 1511-1520.

[26] I.A. Naumov, L.N. Kornilova, D.O. Glukhikh, A.S. Pavlov, E.V. Khabarova, G.A. Ekimovsky and A.V. Vasin, Vestibular function after repeated space flights, Human Physiology 43 (2017), 757-764.

[27] C.M. Oman and M.D. Balkwill, Horizontal angular VOR, nystagmus dumping, and sensation duration in Spacelab SLS-1 crewmember, J Vestib Res 3 (1993), 315-330.

[28] C.M. Oman and M. Kulbaski, Space flight affects the 1-g postrotatory vestibulo-ocular reflex, Adv Otorhino-laryngol 42 (1988), 5-8.

[29] C.M. Oman and H. Weigl, Postflight vestibulo-ocular reflex changes in Space Shuttle/Spacelab D-1 crew, Aviat Space Environ Med 60 (1989), 480-486.

[30] G.D. Paige, Nonlinearity and asymmetry in the human vestibulo-ocular reflex, Acta Otolaryngol (Stockh) 108 (1989), 1-8

[31] G.D. Paige, L. Telford, S.H. Seidman and G.E. Barnes. Human vestibuloocular reflex and its interactions with vision and fixation distance during linear and angular head movement, J Neurophysiol 80 (1998), 2391-2404.

[32] R.J. Peterka, F.O. Black and M.B. Schoenhoff, Age-related changes in human vestibulo-ocular reflexes: Sinusoidal rotation and caloric tests, J Vestib Res 1 (1990), 49-59.

[33] T. Raphan, V. Matsuo and B. Cohen, Velocity storage in the vestibulo-ocular reflex arc (VOR), Exp Brain Res 35 (1979), 229-248.

[34] T. Raphan and B. Cohen, The vestibulo-ocular reflex in three dimensions, Exp Brain Res 145 (2002), 1-27.

[35] M.F. Reschke, E.F. Good and G. Clément, Vestibular symptoms in astronauts following Space Shuttle and International Space Station missions, Otolaryngol Head Neck Surg 1 (2017), 1-8.

[36] M.F. Reschke, O.I. Kolev and G. Clément, Eye-head coordination in 31 Space Shuttle astronauts during visual target 
acquisition, Sci Rep 7 (2017), 14283; doi: 10.1038/s41598017-14752-8

[37] G.W. Snedecor and W.G. Cochran, Statistical Methods, 8th ed., Iowa State University Press, Ames IA, 1989.

[38] W.E. Thornton, W.P. Biggers, W.G. Thomas, S.L. Pool and N.E. Thagard, Electronystagmography and audio potentials in space flight, Laryngoscope 95 (1985), 924-932.

[39] W.E. Thornton, J.J. Uri, T.P. Moore and S.L. Pool, Studies of the horizontal vestibulo-ocular reflex in space flight, Arch Otolaryngol Head Neck Surg 115 (1989), 943-949.

[40] S. Vesterhauge, A. Mansson, T.S. Johansen and K. Zilstorff, Oculomotor response to voluntary head rotations during parabolic flights, The Physiologist 25 (1982), S117-S118.
[41] T. Viéville, G. Clément, F. Lestienne and A. Berthoz, Adaptive modifications of the optokinetic vestibulo-ocular reflexes in microgravity, in: Adaptive Processes in Visual and Oculomotor Systems, E.L. Keller and D.S. Zee, eds., Pergamon Press. New York NY, 1986, pp. 111-120.

[42] E. Viirre, D. Tweed, K. Milner and T.A. Vilis, A reexamination of the gain of the vestibuloocular reflex, J Neurophysiol 56 (1986) 439-450.

[43] D.G.D. Watt, K.E. Money, R.L. Bondar, R.B. Thirsk, M. Garneau and P. Scully-Power, Canadian medical experiments on Shuttle flight 41-G, Can Aeronaut Space J 31 (1985), 215-226. 